\title{
Eine L2-optimale Beobachtertechnik zur Vermeidung von Regler-Windup
}

An L2-optimal Observer Technique for Controller Windup Prevention

Andreas Ortseifen, Jürgen Adamy, Technische Universität Darmstadt

Zusammenfassung Dieser Beitrag stellt eine neue Entwurfsmethodik für Hippes Regler-Antiwindup, das auch als Beobachtertechnik bezeichnet wird, vor. Die Beobachtertechnik basiert auf einer geeigneten Zerlegung des Reglers. Um eine akzeptable Regelgüte sicherzustellen, wurden bislang alle zulässigen Zerlegungen mittels Simulationen evaluiert. Die neu eingeführte Methode bestimmt mittels konvexer Optimierung für jede Zerlegung einen Kennwert, der die Abweichung von dem erwünschten linearen Verhalten des Regelkreises im Sinne der $L_{2}$-Norm charakterisiert. Anhand der Kennwerte wird dann eine Zerlegung gewählt.

\begin{abstract}
$\leadsto$ Summary This article presents a new design method for Hippe's controller anti-windup, also referred to as observer technique. The observer technique prevents controller windup by a suitable decomposition of the controller. So far a decomposition ensuring an acceptable closed-loop performance has been chosen based on simulation results of all valid decompositions. The new method computes a specific value for each decomposition characterizing the degradation from the desired linear performance in terms of the $L_{2}$ norm. Then a proper decomposition is chosen based on these specific values.
\end{abstract}

Schlagwörter Regler-Antiwindup, Beobachtertechnik, $L_{2}$-Verstärkung technique, L2 gain

\section{Einleitung}

Jedes reale Regelsystem ist mit einer Beschränkung der Stellamplitude behaftet. Hält sich das System ausschließlich im zugehörigen begrenzten Bereich des Zustandsraumes auf und werden nur relativ kleine Stellamplituden benötigt, kann die Beschränkung vernachlässigt werden. Es lassen sich dann Regler mittels linearer Entwurfsverfahren entwerfen.

Verlässt das System diesen Bereich, z. B. bei großen Führungsgrößenänderungen oder Störungen, wird die Beschränkung verletzt. Es kann zu erhöhtem Überschwingen, Grenzzyklen oder sogar zu einem instabilen Systemverhalten kommen. Diese Verschlechterung der Regelgüte gegenüber dem linearen Regelkreis wird als Windup bezeichnet.

Um die lokal gut funktionierenden Regler trotzdem einsetzen zu können, existieren Antiwindup-Methoden, die ausschließlich im Sättigungsfall korrigierend eingreifen. Eine Übersicht findet sich in $[7 ; 16]$.
Eine praktikable und einfach anwendbare Methode ist das Antiwindup nach Hippe [4;5]. Die Windup-Effekte werden hierbei in Regler- und Strecken-Windup unterteilt und getrennt voneinander behandelt.

Regler-Windup tritt auf, wenn der Regler instabile Anteile besitzt, die im Sättigungsfall aktiviert werden. Das ist z. B. bei den häufig in der Praxis eingesetzten PI- und PID-Reglern der Fall und führt zu einer Verschlechterung der Regelgüte. Durch eine Zerlegung des Reglers, die als Beobachtertechnik bezeichnet wird, lässt sich der Regler im Sättigungsfall stabilisieren und das Regler-Windup vermeiden.

Alle verbleibenden Windup-Effekte lassen sich der Dynamik der geregelten Strecke zuordnen und werden deshalb als Strecken-Windup bezeichnet. Durch Erweitern des Regelkreises um zusätzliche dynamische Elemente kann Strecken-Windup verhindert werden [5].

Bislang existiert für die Beobachtertechnik kein effizientes, systematisches Verfahren, das die Regelgüte der 
möglichen Zerlegungen des Reglers bewertet. Das in [6] vorgestellte Verfahren der begleitenden Ortskurven basiert auf dem Popov-Kriterium und ist deshalb relativ konservativ und wenig aussagekräftig. Letztlich ist ein Ausprobieren erforderlich, d.h., alle Zerlegungen des Reglers werden mittels Simulationen evaluiert.

Diese Arbeit stellt eine Auswahlmethode für die Beobachtertechnik vor, die ohne aufwendiges Simulieren auskommt. Dabei werden ein Stabilitätskriterium [12] und ein Entwurfsverfahren für AntiwindupMaßnahmen [18] kombiniert und an die Beobachtertechnik angepasst. Für jede Zerlegung des Reglers, die globale asymptotische Stabilität garantiert, wird mittels konvexer Optimierung ein Kennwert bestimmt, der die Regelgüte charakterisiert. Die Auswahl erfolgt dann anhand dieses Kennwertes.

Der Artikel gliedert sich wie folgt: In Abschnitt 2 wird der betrachtete Regelkreis vorgestellt und in Abschnitt 3 die Beobachtertechnik erläutert. Die Stabilität wird in Abschnitt 4 analysiert und die Regelgüte in Abschnitt 5 abgeschätzt. In Abschnitt 6 wird die Auswahlmethode vorgestellt und am Beispiel der Regelung eines hydraulischen Aktors in Abschnitt 7 demonstriert.

\subsection{Hinweise zur Notation}

Der Ausdruck $\boldsymbol{Q} \succ 0$ bedeutet, dass die Matrix $\boldsymbol{Q}$ symmetrisch und positiv definit ist. Analog bedeutet $Q \prec 0$, dass $\boldsymbol{Q}$ symmetrisch und negativ definit ist.

\section{Der betrachtete Regelkreis}

Die lineare, vollständig steuer- und vollständig beobachtbare Strecke der Ordnung $n$ besitzt die Zustandsraumdarstellung

$\dot{\boldsymbol{x}}_{s}=\boldsymbol{A} \boldsymbol{x}_{s}+\boldsymbol{b} u$,

$y=c^{\top} \boldsymbol{x}_{s}$

mit dem Zustandsvektor $\boldsymbol{x}_{s} \in \mathbb{R}^{n}$, dem Eingang $u \in \mathbb{R}$ und dem Ausgang $y \in \mathbb{R}$. Die Übertragungsfunktion ist

$G(s)=\boldsymbol{c}^{\top}(s \boldsymbol{I}-\boldsymbol{A})^{-1} \boldsymbol{b}=\frac{Z(s)}{N(s)}$.

Die Strecke sei stabil, d.h., die Matrix $A$ besitzt ausschließlich Eigenwerte mit negativem Realteil und liege in Regelungsnormalform vor.

Der lineare Regler der Ordnung $k$ besitzt die Zustandsraumdarstellung

$\dot{\boldsymbol{x}}_{r}=\boldsymbol{A}_{r} \boldsymbol{x}_{r}+\boldsymbol{b}_{r} e$,

$u_{r}=\boldsymbol{c}_{r}^{\top} \boldsymbol{x}_{r}+d_{r} e$

mit dem Zustandsvektor $\boldsymbol{x}_{r} \in \mathbb{R}^{k}$, dem Eingang $e \in \mathbb{R}$ und dem Ausgang $u_{r} \in \mathbb{R}$. Die Übertragungsfunktion des Reglers ergibt sich $\mathrm{zu}$

$H(s)=\boldsymbol{c}_{r}^{\top}\left(s \boldsymbol{I}-\boldsymbol{A}_{r}\right)^{-1} \boldsymbol{b}_{r}+d_{r}=\frac{Z_{r}(s)}{N_{r}(s)}$.

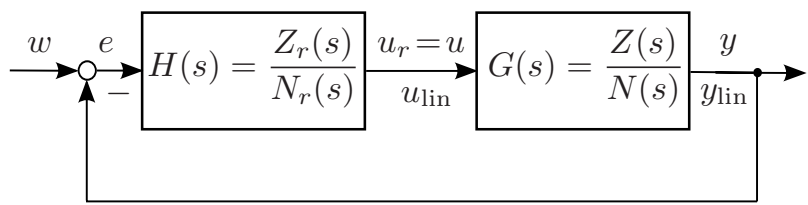

Bild 1 Linearer Regelkreis.

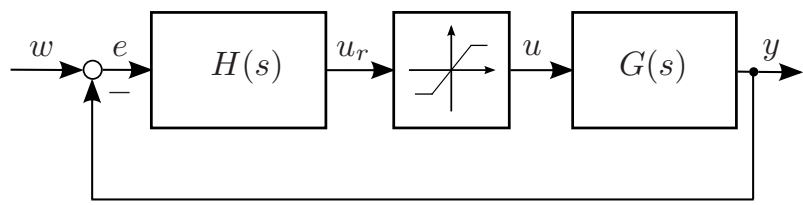

Bild 2 Beschränkter Regelkreis.

Es wird vorausgesetzt, dass der entworfene Regler vorgegebene Dynamikanforderungen erfüllt und zu einem stabilen linearen Regelkreis führt, der in Bild 1 dargestellt ist. Das charakteristische Polynom dieses Regelkreises

$P(s)=Z_{r}(s) Z(s)+N_{r}(s) N(s)$

ist somit ein Hurwitz-Polynom. Um die Linearität hervorzuheben, werden folgende Bezeichnungen eingeführt

$u_{\text {lin }}=u_{r}=u$,

$y_{\text {lin }}=y$.

Für den beschränkten Regelkreis in Bild 2 ist im Sättigungsfall $u \neq u_{r}$. Der Zusammenhang zwischen beiden Größen ist dann durch die Sättigungsfunktion

$u=\operatorname{sat}\left(u_{r}\right)=\operatorname{sgn}\left(u_{r}\right) \min \left(u_{0},\left|u_{r}\right|\right)$

gegeben, mit der maximal möglichen Stellamplitude $u_{0}$.

\section{Vermeidung des Regler-Windup}

Das im Sättigungsfall auftretende Regler-Windup lässt sich vermeiden, indem der Regler (2) wie folgt zerlegt

$H(s)=H_{1}^{-1}(s) \cdot H_{2}(s)=\frac{\Delta(s)}{N_{r}(s)} \cdot \frac{Z_{r}(s)}{\Delta(s)}$

und gemäß Bild 3 realisiert wird [5]. Dabei ist $\Delta(s)$ ein noch zu wählendes Hurwitz-Polynom der Ordnung $k$.

Werden nur die Hurwitz-Polynome $\Delta(s)$ zugelassen, deren Nullstellen gleichzeitig Pole des geschlossenen Regelkreises sind, d.h. Nullstellen des Polynoms $P(s)$,

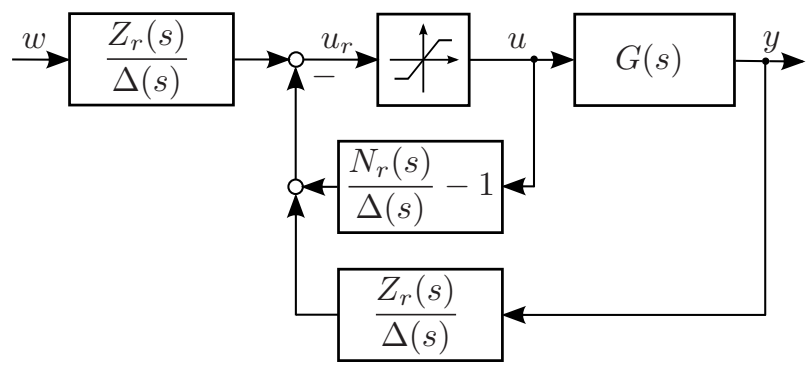

Bild 3 Regler-Antiwindup nach Hippe (Beobachtertechnik). 
so ist sichergestellt, dass ausschließlich Regler-Windup vermieden wird ${ }^{1}$. Diese Vorgehensweise wird auch als Beobachtertechnik bezeichnet. Es lässt sich folgender Satz (in der Formulierung aus [1]) angeben.

Satz 1 (Regler-Antiwindup nach Hippe, [5]). Gegeben sei der beschränkte Regelkreis aus Bild 2 mit dem Regler

$H(s)=\frac{Z_{r}(s)}{N_{r}(s)}$

der Ordnung $k$ und der Regelstrecke

$G(s)=\frac{Z(s)}{N(s)}$

der Ordnung n. Der Regelkreis in Bild 3 besitzt dasselbe lineare Übertragungsverhalten wie der Regelkreis aus Bild 2 und darüber hinaus ein Regler-Antiwindup. Die $k$ Nullstellen des Polynoms $\Delta(s)$ wählt man aus den $n+k$ Nullstellen des charakteristischen Polynoms des linearen unbeschränkten Regelkreises

$P(s)=Z_{r}(s) Z(s)+N_{r}(s) N(s)$.

Beim Entwurf des Regler-Antiwindups müssen die $k$ Nullstellen von $\Delta(s)$ aus den $n+k$ Nullstellen von $P(s)$ gewählt werden. Um eine kompaktere Notation zu erreichen wird dieses Entwurfsproblem auf die Wahl einer Zerlegung des Polynoms $P(s)$ in die zwei Teilpolynome $\Delta(s)$ und $R(s)$ zurückgeführt, d. h.

$P(s)=\Delta(s) R(s)$.

Dabei ist $\Delta(s)$ immer ein Polynom vom Grad $k$ mit reellen Koeffizienten und $R(s)$ ein Polynom vom Grad $n$ mit reellen Koeffizienten.

Eine Zerlegung von $P(s)$ gemäß (8) ist durch ein 2Tupel $\{\Delta(s), R(s)\}$ gegeben. Die Menge

$M_{\mathcal{Z}}=\{\{\Delta(s), R(s)\} \mid \Delta(s) R(s)=P(s)\}$

beinhaltet alle möglichen Zerlegungen des Polynoms $P(s)$. Das können je nach Regler- und Streckenordnung bis zu $\left(\begin{array}{c}n+k \\ k\end{array}\right)$ mögliche Varianten sein ${ }^{2}$.

Wenn die Reglerordnung $k$ ungerade ist und $P(s)$ ausschließlich konjugiert komplexe Nullstellen besitzt, dann existiert keine Zerlegung von $P(s)$ gemäß (8) und es gilt $M_{\mathcal{Z}}=\emptyset$. In diesem seltenen Fall muss $\Delta(s)$ eine reelle Nullstelle besitzen, die sich nach [5] wie folgt bestimmen lässt: Es wird das konjugiert komplexe Nullstellenpaar aus $P(s)$ mit der größten Dämpfung $D$ gewählt. Das zugehörige Polynom $s^{2}+2 D \omega_{0} s+\omega_{0}^{2}$ approximiert man durch $\left(s+\omega_{0}\right)^{2}$ und wählt $\omega_{0}$ als reelle Nullstelle.

Nachfolgend wird nur der Fall $M_{\mathcal{Z}} \neq \emptyset$ betrachtet, also vorausgesetzt, dass Zerlegungen von $P(s)$ existieren. Da

\footnotetext{
${ }^{1}$ Der Regler kann als (reduzierter) Kontrollbeobachter aufgefasst werden und $\Delta(s)$ als charakteristisches Polynom des Beobachters. Eine ausführliche Erklärung findet sich in [1].

${ }^{2}$ Bei einem Regler der Ordnung 2 und einer Strecke der Ordnung 3 ergeben sich bis zu 10 mögliche Zerlegungen.
}

alle Zerlegungen aus $M_{\mathcal{Z}}$ das Regler-Windup vermeiden, besteht völlige Wahlfreiheit. Diese Freiheit kann genutzt werden, um zusätzliche Anforderungen zu erfüllen. Konkret wird folgendes Problem betrachtet:

Problem 1. Wähle eine Zerlegung $\mathcal{Z} \in M_{\mathcal{Z}}$ bzw. die $k$ Nullstellen von $\Delta(s)$ aus den $n+k$ Nullstellen von $P(s)$ so, dass der Regelkreis mit Antiwindup aus Bild 3

a) eine global asymptotisch stabile Ruhelage besitzt und

b) eine Regelgüte aufweist, die möglichst nahe an die des linearen Regelkreises aus Bild 1 heranreicht.

In den nächsten Abschnitten wird eine systematische Methode zur Lösung des Problems vorgestellt.

Bemerkung 1. Die Forderung b) nach möglichst linearer Regelgüte ist ein Ziel vieler Antiwindup-Ansätze, wie beispielsweise [15] und der darin angeführten Referenzen. In Abschnitt 5 wird diese Forderung mathematisch präziser formuliert.

Bemerkung 2. Es können Fälle auftreten, in denen die Vermeidung des Regler-Windup nach Satz 1 nicht ausreicht, um einen stabilen Regelkreis zu garantieren bzw. alle Windup-Effekte auf ein erträgliches Maß zu reduzieren. Noch verbleibende Windup-Effekte lassen sich dann eindeutig auf Strecken-Windup zurückführen und durch entsprechende Maßnahmen beseitigen [4;5].

\section{Stabilitätsanalyse}

Zur Stabilitätsanalyse des in Bild 3 gezeigten Regelkreises wird die Führungsgröße null gesetzt und der Regelkreis in den nichtlinearen Standardregelkreis umgeformt. Das Vorfilter entfällt, da $w=0$ gilt, und die verbleibenden linearen Teile werden in dem System $T(s)$ zusammengefasst. Das Ergebnis ist in Bild 4a dargestellt, wobei der grau hinterlegte lineare Teil $T(s)$ die Übertragungsfunktion

$T(s)=\frac{R(s) \Delta(s)-N(s) \Delta(s)}{N(s) \Delta(s)}=\frac{P(s)}{N(s) \Delta(s)}-1$

besitzt. Dieser lineare Teil ist stabil, da $N(s)$ und $\Delta(s)$ nach Voraussetzung Hurwitz-Polynome sind.

a)

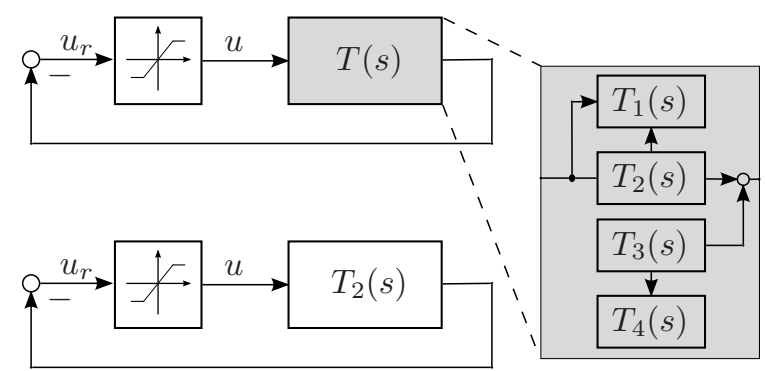

Bild 4 (a) Nichtlinearer Standardregelkreis mit einer Kalman-Zerlegung des linearen Teils. Aus Gründen der Übersichtlichkeit sind nicht alle Verkopplungen zwischen den Teilsystemen dargestellt. (b) Für die Stabilitätsuntersuchung relevantes System. 
Nach obiger Umformung werden in $[4 ; 6]$ das Kreisund das Popov-Kriterium zur Stabilitätsanalyse genutzt. Diese Arbeit setzt auf ein in vielen Fällen weniger konservatives und gleichzeitig einfacher anwendbares Kriterium, das in Abschnitt 4.2 vorgestellt wird. Vorher sind in Abschnitt 4.1 noch einige Überlegungen bezüglich der Steuer- und Beobachtbarkeit des linearen Teils (10) nötig, um die Voraussetzungen für die Anwendung des Kriteriums zu schaffen.

\subsection{Steuer- und Beobachtbarkeit von $T(s)$}

Das Polynom $\Delta(s)$ ist sowohl im Zähler als auch im Nenner der Übertragungsfunktion (10) vorhanden. Außerdem können die Polynome $R(s)$ und $N(s)$ gemeinsame Nullstellen besitzen. Somit ist $T(s)$ nicht vollständig steuerbar und/oder nicht vollständig beobachtbar. Für die weitere Analyse wird das System deshalb in die folgenden Teilsysteme zerlegt (Kalman-Zerlegung [10], siehe Bild 4a):

Teilsystem $T_{1}(s)$ : steuerbar, nicht beobachtbar

Teilsystem $T_{2}(s)$ : steuerbar, beobachtbar

Teilsystem $T_{3}(s)$ : nicht steuerbar, beobachtbar

Teilsystem $T_{4}(s)$ : nicht steuerbar, nicht beobachtbar

Die Teilsysteme 1, 3 und 4 sind stabil und liegen nicht in einem geschlossenen Regelkreis. Folglich spielen Sie für die weitere Stabilitätsuntersuchung keine Rolle. Ausschlaggebend ist das steuer- und beobachtbare Teilsystem 2 mit der stabilen Übertragungsfunktion $T_{2}(s)$, das in einem über die Sättigungsfunktion geschlossenen Kreis liegt. Dieser ist in Bild $4 \mathrm{~b}$ dargestellt und wird durch

$U_{r}(s)=-T_{2}(s) U(s)$,

$u=\operatorname{sat}\left(u_{r}\right)$

beschrieben. Dabei ist

$T_{2}(s)=\frac{R(s)}{N(s)}-1$,

falls die Polynome $R(s)$ und $N(s)$ keine gemeinsamen Nullstellen besitzen. Ansonsten gilt $T_{2}(s)=\hat{R}(s) / \hat{N}(s)-1$, wobei $\hat{R}(s)$ und $\hat{N}(s)$ teilerfremde Polynome sind, die sich nach dem Kürzen der gemeinsamen Nullstellen aus $R(s)$ und $N(s)$ ergeben $^{3}$.

Die Stabilität des Regelkreises aus Bild 3 folgt somit aus der Stabilität des Systems (11), die im nächsten Abschnitt untersucht wird.

\subsection{Stabilitätskriterien}

Die Sättigungsfunktion aus Gl. (11b) ist eine monotone, zeitinvariante Kennlinie, die durch den Ursprung verläuft. Für derartige Kennlinien $u=\Phi\left(u_{r}\right)$ gilt der nachfolgend angegebene Satz.

\footnotetext{
${ }^{3}$ Alle Ergebnisse des Abschnittes 4 bleiben auch in diesem Fall gültig. In den Sätzen und Beweisen ist lediglich $R(s)$ durch $\hat{R}(s)$ und $N(s)$ durch $\hat{N}(s)$ zu ersetzen.
}

Satz 2 (Off-Axis Circle Kriterium, [12]). Gegeben sei der Regelkreis

$U_{r}(s)=-T_{2}(s) U(s)$,

$u=\Phi\left(u_{r}\right)$

mit dem stabilen, vollständig steuer- und beobachtbaren System $T_{2}(s)$. Der Zählergrad von $T_{2}(s)$ sei kleiner gleich dem Nennergrad. Für die zeitinvariante Nichtlinearität $\Phi$ gelte

$\Phi(0)=0$,

$0 \leq \frac{\Phi\left(u_{r, 1}\right)-\Phi\left(u_{r, 2}\right)}{u_{r, 1}-u_{r, 2}} \leq K_{s}$

und zwar für alle $u_{r, 1}, u_{r, 2} \in \mathbb{R}$ und $u_{r, 1} \neq u_{r, 2}$. Wenn die Ortskurve von $T_{2}(s)$ für alle $\omega \geq 0$ rechts einer Geraden mit beliebiger Steigung $q \neq 0$ durch den Punkt $\left(-K_{s}^{-1}, 0\right)$ liegt, dann besitzt das System (13) eine global asymptotisch stabile Ruhelage im Ursprung.

Das Kreiskriterium ist in Satz 2 für $q \rightarrow \infty$ und die betrachtete Klasse an Nichtlinearitäten als Sonderfall enthalten. Der Vorteil gegenüber dem Popov-Kriterium ist die einfache Anwendbarkeit, da nur die Ortskurve von $T_{2}(s)$ benötigt wird. Ausgehend von Satz 2 werden nun zwei Stabilitätskriterien für den Regelkreis mit ReglerAntiwindup aus Bild 3 neu eingeführt. Ein auf Ortskurven basierendes Kriterium ist nachfolgend angegeben.

Satz 3 (Stabilität mittels Ortskurve). Gegeben sei der Regelkreis mit Regler-Antiwindup aus Bild 3 mit der stabilen Strecke $G(s)=Z(s) / N(s)$ gemäß (1), dem Regler (2) und einer Zerlegung $\mathcal{Z}=\{\Delta(s), R(s)\} \in M_{\mathcal{Z}}$ des Polynoms $P(s)$. Die Stellamplitude sei durch die Sättigungsfunktion (6) begrenzt. Wenn die Ortskurve

$T_{2}(j \omega)=\frac{R(j \omega)}{N(j \omega)}-1$

für alle $\omega \geq 0$ vollständig rechts einer Geraden mit beliebiger Steigung $q \neq 0$ durch den Punkt $(-1,0)$ liegt, dann besitzt der Regelkreis eine global asymptotisch stabile Ruhelage im Ursprung.

Beweis. Der Regelkreis aus Bild 3 lässt sich in die Darstellung aus Bild 4a mit $T(s)$ gemäß (10) umformen. Wie in Abschnitt 4.1 gezeigt, reduziert sich die Stabilitätsuntersuchung dieses Regelkreises auf das vollständig steuer- und beobachtbare Teilsystem (11), dessen Zählergrad immer kleiner gleich dem Nennergrad ist. Die Sättigungsfunktion (6) erfüllt die Bedingungen (14) und insbesondere (15) mit $K_{s}=1$. Deshalb lässt sich Satz 2 anwenden, woraus Satz 3 direkt folgt.

Aufgrund der Struktur von $T_{2}(j \omega)$ muss nicht die komplette Ortskurve betrachtet werden. Es genügt, den Phasenverlauf von $T_{2}(j \omega)+1 \mathrm{zu}$ analysieren, wie in dem folgenden Kriterium angegeben. 
Satz 4 (Phasenbedingung für Stabilität). Gegeben sei der Regelkreis mit Regler-Antiwindup aus Bild 3 mit der stabilen Strecke $G(s)=Z(s) / N(s)$ gemäß (1), dem Regler (2) und einer Zerlegung $\mathcal{Z}=\{\Delta(s), R(s)\} \in M_{\mathcal{Z}}$. Die Stellamplitude sei durch die Sättigungsfunktion (6) begrenzt. Wenn ein $\delta \in\left[-90^{\circ}+\epsilon, 90^{\circ}-\epsilon\right]$ mit beliebig kleinem $\epsilon>0$ existiert, sodass

$-90^{\circ}-\delta<\arg \left\{\frac{R(j \omega)}{N(j \omega)}\right\}<90^{\circ}-\delta \quad \forall \omega \geq 0$

gilt, dann besitzt der Regelkreis eine global asymptotisch stabile Ruhelage im Ursprung.

Beweis. Nach Satz 3 besitzt der Regelkreis mit ReglerAntiwindup aus Bild 3 eine global asymptotisch stabile Ruhelage, wenn die Ortskurve des Frequenzganges $T_{2}(j \omega)$ rechts einer Geraden durch den Punkt $(-1,0)$ mit beliebiger Steigung $q \neq 0$ liegt. Eine äquivalente Forderung ist, dass die Ortskurve von $T_{2}(j \omega)+1$ rechts einer Ursprungsgeraden mit Steigung $q \neq 0$ bzw. dem Steigungswinkel $\alpha=\arctan (q)$ liegt, wie in Bild 5a exemplarisch dargestellt. Diese Forderung lässt sich in zwei Bedingungen an Betrag und Phase der Ortskurve $T_{2}(j \omega)+1$ umwandeln. Erstens muss für den Betrag

$\left|T_{2}(j \omega)+1\right|=\frac{|R(j \omega)|}{|N(j \omega)|}>0 \quad \forall \omega \geq 0$

gelten ${ }^{4}$. Das ist immer erfüllt, da $R(s)$ und $N(s)$ HurwitzPolynome sind und den gleichen Grad besitzen. Um dies zu zeigen, wird das Lückenkriterium benötigt.

Lemma 1 (Lückenkriterium, nach [13]). Das Polynom

$R(s)=s^{n}+a_{n-1} s^{n-1}+\ldots+a_{1} s+a_{0}$

hat genau dann nur Nullstellen mit negativem Realteil, wenn die Gleichungen $\operatorname{Re}\{R(j \omega)\}=0$ und $\operatorname{Im}\{R(j \omega)\}=0$ für $0 \leq \omega \leq \infty$ zusammen $n$ reelle Nullstellen haben und die Nullstellen der Polynome $\operatorname{Re}\{R(j \omega)\}$ und $\operatorname{Im}\{R(j \omega)\}$ abwechselnd aufeinander folgen.

Folglich gilt für ein Hurwitz-Polynom

$|R(j \omega)|^{2}=\operatorname{Re}\{R(j \omega)\}^{2}+\operatorname{Im}\{R(j \omega)\}^{2}>0$

für alle $\omega \geq 0$, da nach dem Lückenkriterium nie Realund Imaginärteil gleichzeitig null werden können. So kann der Betrag der Ortskurve aus Gl. (18) nur dann null werden, wenn $|N(j \omega)|$ schneller gegen einen unendlichen Wert strebt als $|R(j \omega)|$. Da beide Polynome den gleichen Grad besitzen ist auch das nicht der Fall.

Zweitens muss für die Phase der Ortskurve $T_{2}(j \omega)+1$ gelten: Der maximale Phasenwinkel ist kleiner als der Steigungswinkel $\alpha=\arctan (q)$ der Ursprungsgeraden

\footnotetext{
${ }^{4}$ Dürfte der Betrag auch null werden, so würde die Ortskurve die Ursprungsgerade im Ursprung berühren oder schneiden.
}

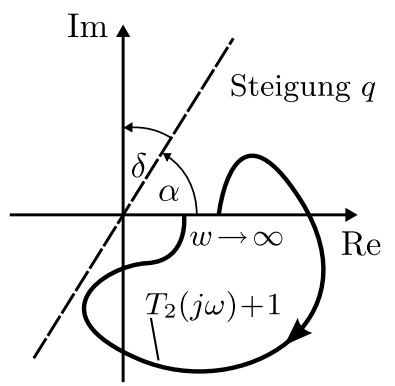

a)

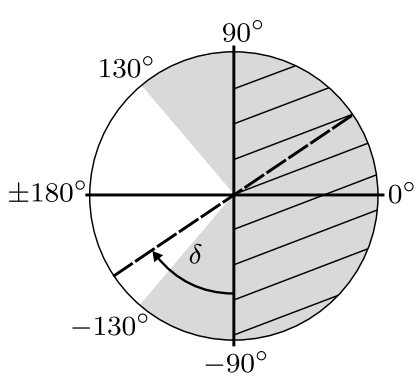

b)
Bild 5 (a) Die Ortskurve von $T_{2}(j \omega)+1$ liegt, wie in Satz 4 gefordert, rechts einer Ursprungsgeraden mit Steigung $q$ bzw. dem Steigungswinkel $\alpha=\arctan (q)$. (b) Vergleich der zulässigen Phasenwinkel verschiedener Kriterien für die Ortskurve $T_{2}(j \omega)+1$ : Phasenkriterium (grau), Kreiskriterium (schraffiert) und Satz 4 (Bereich rechts der gestrichelten Gerade).

und der minimale Phasenwinkel ist größer als $\alpha-180^{\circ}$, d. h.

$\alpha-180^{\circ}<\arg \left\{\frac{R(j \omega)}{N(j \omega)}\right\}<\alpha \quad \forall \omega \geq 0$.

Nur dann liegt die Ortskurve komplett in der rechten Hälfte der durch die Gerade geteilten komplexen Ebene. Aus Bild 5a ergibt sich

$\alpha=\arctan (q)=90^{\circ}-\delta$.

Einsetzen von Gl. (20) in Ungl. (19) liefert Ungl. (17). Aus Gl. (20) folgt weiter $q=\tan \left(90^{\circ}-\delta\right)$. Da $q=0$ nach Satz 2 nicht zulässig ist, darf $\delta$ nur aus $\left[-90^{\circ}+\epsilon, 90^{\circ}-\epsilon\right]$ mit einem beliebig kleinen $\epsilon>0$ gewählt werden.

Das Stabilitätskriterium aus Satz 4 ähnelt in seiner Struktur dem empirischen Phasenkriterium aus [5]. Dieses Phasenkriterium macht eine qualitative Aussage über die Regelgüte: Wenn der lineare Regelkreis aus Bild 1 gut gedämpft ausgelegt ist, regt ein Ansprechen der Stellbegrenzung des Regelkreises mit Antiwindup aus Bild 3 keine erhöhte Schwingneigung an, wenn

$-130^{\circ}<\arg \left\{\frac{R(j \omega)}{N(j \omega)}\right\}<130^{\circ} \quad \forall \omega \geq 0$

gilt. In Bild 5b sind für verschiedene Kriterien die zulässigen Bereiche für den Phasenwinkel der Ortskurve $T_{2}(j \omega)+1$ dargestellt. Das Kreiskriterium garantiert eine global asymptotisch stabile Ruhelage, beschränkt den Phasenwinkel aber auf $\pm 90^{\circ}$ (schraffierter Bereich). Das empirische Phasenkriterium bietet einen größeren Winkelbereich (grau), gibt aber keine Stabilitätsgarantie. Satz 4 erlaubt einen flexibel einstellbaren Winkelbereich von $180^{\circ}$ (Bereich rechts der gestrichelten Gerade) und garantiert globale Stabilität.

\section{Regelgüte}

In diesem Abschnitt wird der Einfluss einer Zerlegung $\mathcal{Z} \in M_{\mathcal{Z}}$ auf die Regelgüte untersucht. Der Regelkreis mit Regler-Antiwindup weicht im Sättigungsfall, je 
nach gewählter Zerlegung des Polynoms $P(s)=\Delta(s) R(s)$, unterschiedlich stark von dem erwünschten linearen Verhalten ab. Diese Diskrepanz (engl. Mismatch) kann mithilfe der sogenannten Mismatch-Darstellung des Regelkreises $[14 ; 15 ; 18 ; 19]$ quantifiziert und in einen interpretierbaren Kennwert abgebildet werden.

\subsection{Mismatch-Darstellung}

Der Regelkreis mit Antiwindup aus Bild 3 lässt sich in die Form aus Bild 6 überführen [19]. Diese MismatchDarstellung besitzt dasselbe Übertragungsverhalten, teilt aber das ursprüngliche System in den linearen Regelkreis aus Bild 1 und den grau hinterlegten nichtlinearen Teil auf.

Der Vorteil dieser Darstellung besteht darin, dass der Ausgang des nichtlinearen Teils durch

$y_{d}=y_{\text {lin }}-y$

gegeben ist und somit die Differenz zwischen dem linearen Verhalten $y_{\text {lin }}$ und dem Ausgang $y$ des Regelkreises mit Antiwindup charakterisiert. Im Sättigungsfall überschreitet $\left|u_{\text {lin }}\right|$ die maximal mögliche Stellamplitude $u_{0}$. Der nichtlineare Teil wird angeregt und $y_{d} \neq 0$.

In [19] ist eine allgemeine Zustandsraumdarstellung des nichtlinearen Teils der Mismatch-Darstellung angegeben. Mit dem Zustandsvektor $z \in \mathbb{R}^{n}$ lässt sich diese wie folgt schreiben

$\dot{z}=A_{f} z+\boldsymbol{b} \operatorname{dzn}\left(u_{\operatorname{lin}}-f^{\top} z\right)$,

$y_{d}=c^{\top} z$

mit $\boldsymbol{A}_{f}=\boldsymbol{A}+\boldsymbol{b} \boldsymbol{f}^{\top}$. Dabei sind $\boldsymbol{A}, \boldsymbol{b}, \boldsymbol{c}$ die Systemmatrizen der Strecke in Regelungsnormalform und der Operator $\mathrm{dzn}(\cdot)$ steht für die Totzone

$\tilde{u}=\operatorname{dzn}\left(u_{r}\right)=u_{r}-\operatorname{sat}\left(u_{r}\right)$.

Der Vektor $f \in \mathbb{R}^{n}$ beeinflusst das Verhalten des Antiwindups. In $[14 ; 15 ; 18 ; 19]$ ist $f$ zunächst nicht bekannt und wird mittels verschiedener Verfahren entworfen. In dieser Arbeit wird die Mismatch-Darstellung nicht

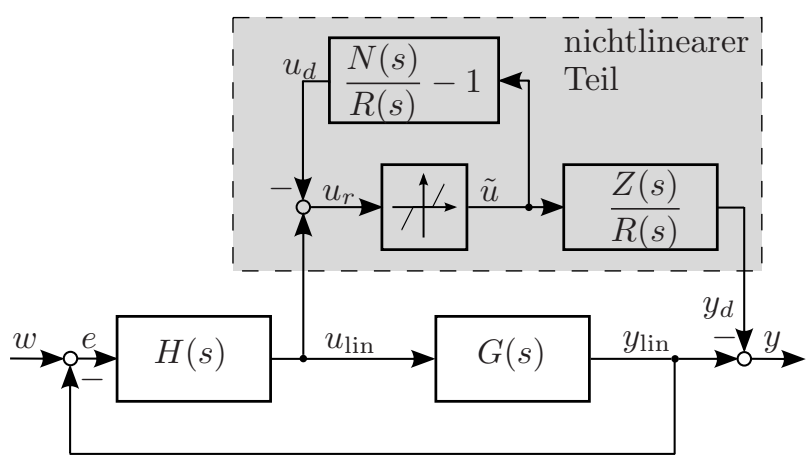

Bild 6 Mismatch-System: Eine alternative Darstellung des Regelkreises mit Regler-Antiwindup. Das Ein-/Ausgangsverhalten ist identisch mit dem Regelkreis in Bild 3. Die Totzone ist in Bild 7a detailliert dargestellt. zum Entwurf, sondern zur Analyse der Reglerzerlegungen des Antiwindups nach Hippe verwendet. Im Anhang wird gezeigt. dass $f$ dann je nach gewählter Zerlegung $\mathcal{Z}=\{\Delta(s), R(s)\} \in M_{\mathcal{Z}}$ durch

$\operatorname{det}\left(s \boldsymbol{I}-\boldsymbol{A}-\boldsymbol{b} \boldsymbol{f}^{\top}\right)=R(s)$

definiert ist.

Ein geeigneter Kennwert für die Abweichung des Regelkreises mit Antiwindup vom linearen Verhalten ist die Verstärkung des nichtlinearen Teils vom Eingang $u_{\text {lin }}$ zum Ausgang $y_{d}$. Diese ist allgemein gegeben durch

$$
\sup _{\left\|u_{\operatorname{lin}}\right\|_{i} \neq 0} \frac{\left\|y_{d}\right\|_{i}}{\left\|u_{\operatorname{lin}}\right\|_{i}},
$$

wobei $\|\cdot\|_{i}$ für eine beliebige Norm steht. Hier wird, wie in $[14 ; 15]$, die $L_{2}$-Norm

$\left\|y_{d}\right\|_{L_{2}}=\sqrt{\int_{0}^{\infty} y_{d}(t)^{2} d t}<\infty$

verwendet, die ein Maß für die Energie des Signals ist. Mit der $L_{2}$-Norm lässt sich die $L_{2}$-Verstärkung definieren.

Definition 1 ( $\boldsymbol{L}_{2}$-Verstärkung, [3]). Das System (23) mit $z(0)=\mathbf{0}$ hat eine endliche $L_{2}$-Verstärkung kleiner gleich $\gamma$, wenn

$$
\sup _{\left\|u_{\operatorname{lin}}\right\|_{L_{2}} \neq 0} \frac{\left\|y_{d}\right\|_{L_{2}}}{\left\|u_{\operatorname{lin}}\right\|_{L_{2}}} \leq \gamma
$$

Eine möglichst lineare Regelgüte lässt sich durch die Wahl eines $\mathcal{Z} \in M_{\mathcal{Z}}$ erreichen, das eine möglichst kleine obere Schranke $\gamma$ für die $L_{2}$-Verstärkung des Systems (23) garantiert. Dann ist die Abweichung des Systemausganges $y$ von dem gewünschten linearen Verhalten $y_{\text {lin }}$ klein im Sinne der $L_{2}$-Norm $[14 ; 15]$.

\subsection{Abschätzung der $L_{2}$-Verstärkung}

Zur Abschätzung der $L_{2}$-Verstärkung des Systems (23) lässt sich, wie in $[14 ; 15]$, das Konzept der Dissipativität ${ }^{5}$ nutzen. Im Weiteren wird der folgende Zusammenhang benötigt.

Lemma 2 ( $\boldsymbol{L}_{2}$-Verstärkung, nach [3]). Das System (23) hat eine $L_{2}$-Verstärkung kleiner gleich $\gamma>0$, wenn eine positiv definite Funktion $V(z)$, die bezüglich $z$ stetig differenzierbar ist, existiert, sodass die folgende Dissipativitätsungleichung für alle $z, u_{\mathrm{lin}}, y_{d}$ erfüllt ist

$\dot{V}(\boldsymbol{z}) \leq \gamma^{2}\left\|u_{\text {lin }}\right\|_{2}^{2}-\left\|y_{d}\right\|_{2}^{2}$.

Dabei ist $\dot{V}(\boldsymbol{z})=\dot{\boldsymbol{z}}^{\top} \operatorname{grad} V(\boldsymbol{z})$. Mit Lemma 2 lässt sich eine obere Schranke für die $L_{2}$-Verstärkung des Systems (23) wie folgt abschätzen.

\footnotetext{
${ }^{5}$ Eine Einführung findet sich in $[3 ; 8]$.
} 
Satz 5 (Abschätzung der $L_{2}$-Verstärkung). Eine obere Schranke $\gamma>0$ für die $L_{2}$-Verstärkung des Systems (23) mit dem durch Gl. (25) definierten $f$ existiert, wenn sich eine Matrix $Q \succ 0$, ein $\tau>0$ und ein $\mu>0$ für ein vorher gewähltes $K \geq 1$ finden lassen, sodass die folgende lineare Matrixungleichung erfüllt ist

$$
\left[\begin{array}{ccc}
\boldsymbol{A}_{f}^{\top} \boldsymbol{Q}+\boldsymbol{Q} A_{f}+\boldsymbol{c} \boldsymbol{c}^{\top} & \boldsymbol{Q b}-\boldsymbol{f K \tau} & \mathbf{0} \\
\boldsymbol{b}^{\top} \boldsymbol{Q}-\boldsymbol{f}^{\top} K \tau & -2 \tau & K \tau \\
\mathbf{0} & K \tau & -\mu
\end{array}\right] \prec 0 .
$$

Die obere Schranke ist dann gegeben durch $\gamma=\sqrt{\mu}$.

Beweis. Der Beweis von Satz 5 ist implizit in dem sehr knapp formulierten Beweis des Entwurfssatzes 1 aus [18] enthalten. Da dort einige zum Verständnis wichtige Zwischenschritte fehlen, ist er hier in einer ausführlicheren Form angegeben.

Das nichtlineare System (23) hat gemäß Lemma 2 eine endliche $L_{2}$-Verstärkung kleiner gleich $\gamma>0$, wenn die Bedingung (29) erfüllt ist. Das trifft $\mathrm{zu}$, wenn für alle $\left[\begin{array}{lll}z & u_{\text {lin }} & y_{d}\end{array}\right] \neq \mathbf{0}$ die Ungleichung

$\dot{z}^{\top} \operatorname{grad} V(\boldsymbol{z})<\gamma^{2}\left\|u_{\text {lin }}\right\|_{2}^{2}-\left\|y_{d}\right\|_{2}^{2}$

gilt. Für den Fall $\left[\begin{array}{lll}z & u_{\operatorname{lin}} & y_{d}\end{array}\right]=\mathbf{0}$ gilt immer

$\dot{z} \operatorname{grad} V(z)=\gamma^{2}\left\|u_{\text {lin }}\right\|_{2}^{2}-\left\|y_{d}\right\|_{2}^{2}=0$,

sodass dieser nicht weiter betrachtet werden muss. Wählt man $V(z)=z^{\top} \mathbf{Q} z$ mit einer positiv definiten Matrix $Q \in \mathbb{R}^{n \times n}$ und setzt die Systemgleichungen (23) in (31) ein, ergibt sich

$z^{\top}\left(A_{f}^{\top} Q+Q A_{f}+c c^{\top}\right) z+$

$\tilde{u}^{\top} \boldsymbol{b}^{\top} \mathbf{Q} z+\boldsymbol{z}^{\top} \mathbf{Q} \boldsymbol{b} \tilde{u}-\gamma^{2} u_{\operatorname{lin}}^{\top} u_{\operatorname{lin}}<0$.

Dabei ist $\tilde{u}$ für jedes $z$ und $u_{\text {lin }}$ eindeutig durch

$\tilde{u}=\operatorname{dzn}\left(u_{r}\right)=\operatorname{dzn}\left(u_{\operatorname{lin}}-f^{\top} z\right)$

definiert. Lässt sich ein positiv definites $\boldsymbol{Q}$ finden, sodass die Ungleichung (33) für ein $\gamma>0$, alle $\left[z u_{\text {lin }}\right] \neq \mathbf{0}$ und die durch (34) definierten zugehörigen $\tilde{u}$ erfüllt ist, dann hat das System (23) eine endliche $L_{2}$-Verstärkung.

Aufgrund der Totzone ist die Nebenbedingung (34) schwierig zu behandeln. Deshalb wird die implizite Nebenbedingung

$\tilde{u} \tilde{u} \leq u_{r} \tilde{u} K=\left(u_{\operatorname{lin}}-f^{\top} z\right) \tilde{u} K$

mit $K \in \mathbb{R}$ verwendet, die eine Menge zulässiger $\tilde{u}$ für jedes $z$ und $u_{\text {lin }}$ bzw. jedes $u_{r}=u_{\text {lin }}-f^{\top} z$ definiert. Grafisch entspricht diese Bedingung dem in Bild 7a grau hinterlegten Sektor $[0, K]$ in der $u_{r}-\tilde{u}$-Ebene. Für $K \geq 1$ schließt der Sektor die Totzone, d.h. Bedingung (34), ein. Daraus folgt: Gilt die Ungleichung (33) unter der Nebenbedingung (35) mit $K \geq 1$ für ein $\gamma>0$ und alle $\left[\begin{array}{ll}z & u_{\text {lin }}\end{array}\right] \neq \mathbf{0}$, dann gilt sie auch unter der weniger restriktiven Nebenbedingung (34).

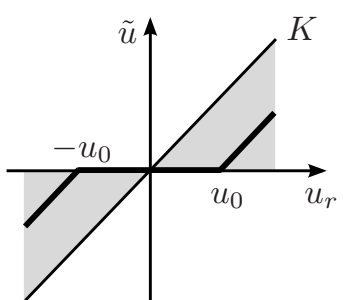

a)

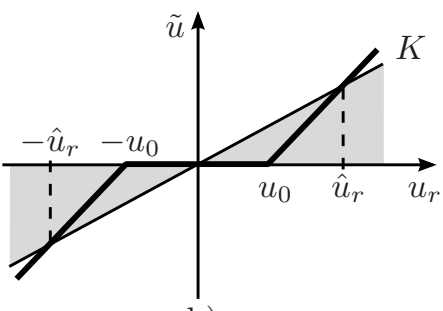

b)
Bild 7 (a) Die Totzone aus Gl. (24) liegt in einem Sektor $[0, K]$ mit $K \geq 1$. (b) Lokal, d. h. für Eingangsgrößen $-\hat{u}_{r} \leq u_{r} \leq \hat{u}_{r}$, liegt die Totzone in einem Sektor $[0, K]$ mit $K<1$.

Der Rest des Beweises zeigt die Herleitung der Matrixungleichung (30) aus der Ungleichung (33) und der Nebenbedingung (35). Multiplizieren der Bedingung (35) mit zwei und anschließendes Ausmultiplizieren ergibt

$2 u_{\operatorname{lin}} \tilde{u} K-2 f^{\top} z \tilde{u} K-2 \tilde{u} \tilde{u} \geq 0$.

Diese Ungleichung lässt sich mit $\boldsymbol{\xi}=\left[\begin{array}{lll}\boldsymbol{z} & \tilde{u} & u_{\operatorname{lin}}\end{array}\right]^{\top}$ darstellen als

$\xi^{\top} M_{2} \xi \geq 0$,

wobei $\boldsymbol{M}_{2}=\left[\begin{array}{ccc}\mathbf{0} & -\boldsymbol{f} K & \mathbf{0} \\ -\boldsymbol{f}^{\top} K & -2 & K \\ \mathbf{0} & K & 0\end{array}\right]$.

Die Ungleichung (33) lässt sich umformen in

$\xi^{\top} M_{1} \xi<0$,

wobei $\boldsymbol{M}_{1}=\left[\begin{array}{ccc}\boldsymbol{A}_{f}^{\top} \boldsymbol{Q}+\boldsymbol{Q} \boldsymbol{A}_{f}^{\top}+\boldsymbol{c} \boldsymbol{c}^{\top} & \boldsymbol{Q} \boldsymbol{b} & \mathbf{0} \\ \boldsymbol{b}^{\top} \boldsymbol{Q} & 0 & 0 \\ \mathbf{0} & 0 & -\gamma^{2}\end{array}\right]$.

Mit der S-Prozedur [2;14] können die Bedingung (38) und die Nebenbedingung (37) in eine einzige Ungleichung überführt werden. Wenn eine Konstante $\tau>0$ existiert, sodass

$M_{1}+\tau M_{2} \prec 0$

erfüllt ist, dann gilt

$\xi^{\top} \boldsymbol{M}_{2} \xi \geq 0 \Rightarrow \xi^{\top} \boldsymbol{M}_{1} \xi<0$.

Das bedeutet: Ist die Ungleichung (39) für ein positiv definites $Q$, ein $\gamma>0$, ein $\tau>0$ und ein ausgewähltes $K \geq 1$ erfüllt, dann gilt Ungleichung (38) für alle $\left[\begin{array}{ll}z & u_{\operatorname{lin}}\end{array}\right] \neq \mathbf{0}$ und alle $\tilde{u}$, die Ungleichung (37) erfüllen. Die Ungleichung (39) entspricht nach der Substitution $\mu=\gamma^{2}$ der Ungleichung (30) aus Satz 5.

Es kann der Fall eintreten, dass die Matrixungleichung (30) für eine Zerlegung $\mathcal{Z} \in M_{\mathcal{Z}}$ nicht erfüllbar und somit Satz 5 nicht anwendbar ist. Zur Einschätzung der Regelgüte kann in solchen Fällen eine obere Schranke $\gamma$ der lokalen $L_{2}$-Verstärkung bzw. der $L_{2}$ Kleinsignalverstärkung bestimmt werden [18]. Diese obere Schranke ist nur für kleine Signale $u_{\text {lin }}$ gültig und stellt somit eine lokale Abschätzung der Regelgüte dar. 
Satz 6 (Lokale $L_{2}$-Verstärkung). Eine obere Schranke für die lokale $L_{2}$-Verstärkung $\gamma>0$ des Systems (23) mit dem durch Gl. (25) definierten $f$ existiert, wenn sich eine Matrix $Q \succ 0$, ein $\tau>0$ und ein $\mu>0$ für ein vorher gewähltes $K \in(0,1)$ finden lassen, sodass die Matrixungleichung (30) erfüllt ist. Die obere Schranke ist dann gegeben durch $\gamma=\sqrt{\mu}$.

Beweis. Der Beweis verläuft analog zu dem Beweis von Satz 5. Allerdings muss die Ungleichung (35) nicht für alle $u_{r}$ überprüft werden. Wenn nur beschränkte Eingangssignale $u_{\text {lin }}$ zulässig sind und ein stabiles System (23) vorliegt, dann kann das Argument $u_{r}=u_{\text {lin }}-\boldsymbol{f} z$ der Totzone nicht beliebig groß werden, d.h. $\left|u_{r}\right|<\hat{u}_{r}$. Die Totzone lässt sich deshalb lokal in einen Sektor $[0, K]$ mit $0<K<1$ einschließen, wie in Bild 7b dargestellt. Deshalb sind in der linearen Matrixungleichung (30) für $K$ auch Werte im Intervall $(0,1)$ zulässig.

\section{Die Auswahlmethode}

Mithilfe der bisherigen Ergebnisse wird die Auswahlmethode zur Lösung des in Abschnitt 3 definierten Problems formuliert. Bei der Auswahl eines $\mathcal{Z} \in M_{\mathcal{Z}}$, das einen stabilen Regelkreis und eine möglichst lineare Regelgüte garantiert, geht man in zwei Schritten vor:

Schritt 1: Mittels Satz 3 oder 4 wird die Stabilität des Regelkreises mit Antiwindup für alle $\mathcal{Z} \in M_{\mathcal{Z}}$ überprüft. Die Zerlegungen, die zu einem global asymptotisch stabilen Regelkreis führen, werden in der Menge $M_{\mathcal{Z}}^{\text {stabil }}$ zusammengefasst.

Schritt 2: Mittels Satz 5 wird für jedes $\mathcal{Z} \in M_{\mathcal{Z}}^{\text {stabil }}$ ein Kennwert $^{6}$ für die Abweichung von der linearen Regelgüte abgeschätzt. Dazu wird für $K=1$ das Optimierungsproblem ${ }^{7}$

$$
\min \mu
$$

unter der Nebenbedingung (30)

in den Variablen $\mu, \boldsymbol{Q}$ und $\tau$ gelöst. So wird jedem $\mathcal{Z} \in M_{\mathcal{Z}}^{\text {stabil }}$ ein $\gamma=\sqrt{\mu}$ zugeordnet. Ausgewählt wird dann die Zerlegung mit dem kleinsten $\gamma$.

Falls sich aus dem Optimierungsproblem (41) nicht für jedes $\mathcal{Z} \in M_{\mathcal{Z}}^{\text {stabil }}$ eine Lösung ergibt, wird Schritt 2 für ein möglichst großes $K<1$ und alle $\mathcal{Z} \in M_{\mathcal{Z}}^{\text {stabil }}$ wiederholt. Der Kennwert ist dann nach Satz 6 eine obere Schranke der $L_{2}$-Kleinsignalverstärkung des Systems (23).

\section{Beispiel: Hydraulischer Aktor}

Es wird der hydraulische Aktor aus [9] betrachtet, dessen schematischer Aufbau in Bild 8 dargestellt ist. Die Eingangsspannung $u$ wird über einen Spannungs-DruckWandler in den Druck $p_{1}$ gewandelt. Dieser Druck bewirkt über ein Rohr einen Volumenstrom $\dot{v}$ in den

\footnotetext{
${ }^{6}$ Der Kennwert ist eine obere Schranke der $L_{2}$-Verstärkung des Systems (23).

${ }^{7}$ Für Probleme dieser Art stehen sehr schnelle und zuverlässige Lösungsalgorithmen zur Verfügung $[11 ; 17]$.
}

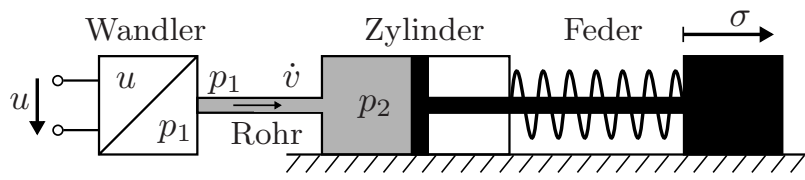

Bild 8 Hydraulischer Aktor.

Zylinder. Der Zylinderkolben ist mit einer Masse verbunden. Die Auslenkung der Masse wird mit $\sigma$ bezeichnet, ihre Geschwindigkeit mit $\dot{\sigma}$. Mit den Zuständen $x_{1, s}=\sigma$ in $\mathrm{cm}, x_{2, s}=\dot{\sigma}$ in $\mathrm{cm} / \mathrm{s}$ und $x_{3, s}=p_{1}$ in $\mathrm{N} / \mathrm{cm}^{2}$ ergibt sich das lineare Zustandsraummodell

$\dot{\boldsymbol{x}}_{s}=\left[\begin{array}{ccc}0 & 1 & 0 \\ -10 & -1,167 & 25 \\ 0 & 0 & -0,8\end{array}\right] \boldsymbol{x}_{s}+\left[\begin{array}{c}0 \\ 0 \\ 2,4\end{array}\right] u$,

$y=\left[\begin{array}{lll}1 & 0 & 0\end{array}\right] \boldsymbol{x}_{s}$.

Die Systemmatrix $\boldsymbol{A}$ hat die Eigenwerte $\lambda_{1}=-0,8$ und $\lambda_{2,3}=-0,584 \pm j 3,108$. Die Stellamplitude ist durch die Sättigungsfunktion nach Gl. (6) mit $u_{0}=10,5 \mathrm{~V}$ begrenzt.

Zur Regelung kommt ein PID-Regler mit phasenanhebendem Korrekturglied zum Einsatz. Der PID-Regler kompensiert dabei das konjugiert komplexe Polpaar der Strecke. Die Übertragungsfunktion des Reglers lautet

$H(s)=\frac{17,764\left(s^{2}+1,167 s+10\right)}{s(s+100)} \frac{(s+0,731)}{(s+5)}$.

Dieser Entwurf garantiert im linearen Bereich, dass maximal 1\% Überschwingen auftritt. Das charakteristische Polynom $P(s)$ des linearen Regelkreises besitzt die Nullstellen

$v_{1,2}=-0,58 \pm j 3,11, \quad v_{3}=-0,70$,

$v_{4,5}=-2,49 \pm j 2,20, \quad v_{6}=-100,11$.

Ein Regler-Windup soll durch Satz 1 vermieden werden. Die vier Zerlegungen von $P(s)$ werden im Weiteren mit $\mathcal{Z}_{i}=\left\{\Delta_{i}(s), R_{i}(s)\right\}$ für $i=1, \ldots, 4$ bezeichnet. Tabelle 1 gibt die Nullstellen der Polynome $\Delta_{i}(s)$ und $R_{i}(s)$ an.

Die vier möglichen Zerlegungen von $P(s)$ werden in der Menge $M_{\mathcal{Z}}=\left\{\mathcal{Z}_{1}, \mathcal{Z}_{2}, \mathcal{Z}_{3}, \mathcal{Z}_{4}\right\}$ zusammengefasst. Für den Entwurf wird die Auswahlmethode aus Abschnitt 6 verwendet:

Schritt 1: Aus den Phasenverläufen in Bild 9 lässt sich erkennen, dass alle $\mathcal{Z} \in M_{\mathcal{Z}}$ die Phasenbedingung aus Satz 4 einhalten. Globale asymptotische Stabilität kann somit für alle $\mathcal{Z} \in M_{\mathcal{Z}}$ nachgewiesen werden, d. h. $M_{\mathcal{Z}}=M_{\mathcal{Z}}^{\text {stabil }}$

Tabelle 1 Mögliche Zerlegungen von $P(s)$ und die geschätzten oberen Schranken der lokalen $L_{2}$-Verstärkungen für $K=0,97$.

\begin{tabular}{lcccc}
\hline & $\mathcal{Z}_{1}$ & $\mathcal{Z}_{2}$ & $\mathcal{Z}_{3}$ & $\mathcal{Z}_{4}$ \\
\hline$\Delta_{i}(s)$ & $v_{3}, v_{4}, v_{5}$ & $v_{1}, v_{2}, v_{3}$ & $v_{4}, v_{5}, v_{6}$ & $v_{1}, v_{2}, v_{6}$ \\
$R_{i}(s)$ & $v_{1}, v_{2}, v_{6}$ & $v_{4}, v_{5}, v_{6}$ & $v_{1}, v_{2}, v_{3}$ & $v_{3}, v_{4}, v_{5}$ \\
$\gamma$ & 4,15 & 1,58 & 7,30 & 8,61 \\
\hline
\end{tabular}




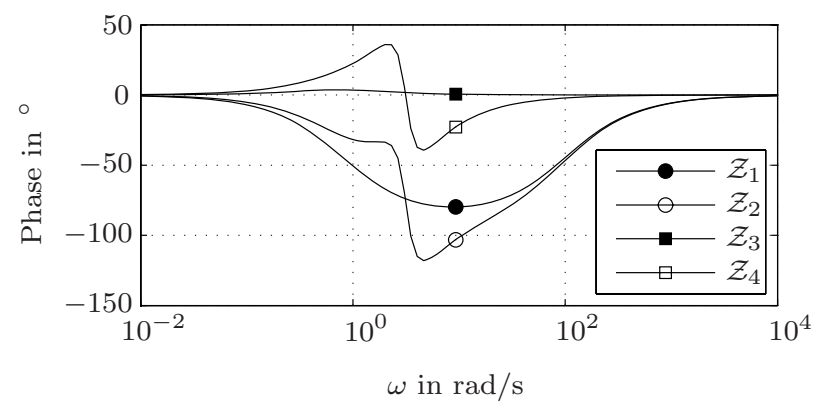

Bild 9 Phasenverläufe $\arg \left\{R_{i}(j \omega) / N(j \omega)\right\}$ für $i=1, \ldots, 4$ der vier Zerlegungen $\mathcal{Z}_{1}, \mathcal{Z}_{2}, \mathcal{Z}_{3}, \mathcal{Z}_{4}$ von $P(s)$. Alle Zerlegungen führen nach Satz 4 zu einem Regelkreis mit einer global asymptotisch stabilen Ruhelage.

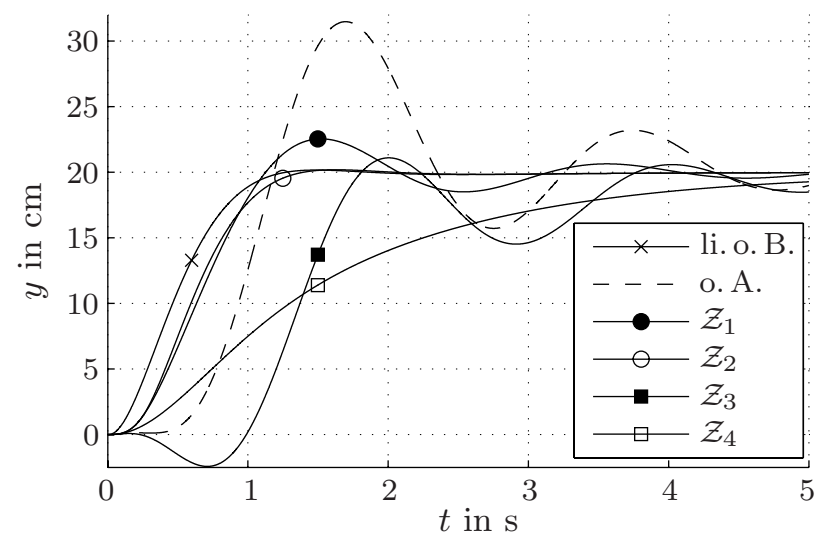

Bild 10 Antwort des hydraulischen Aktors auf einen Sollwertsprung von $20 \mathrm{~cm}$. Dargestellt ist die Ausgangsgröße $y$ für $\mathcal{Z}_{1}$ bis $\mathcal{Z}_{4}$, das lineare geregelte System ohne Beschränkung (li. o. B.) aus Bild 1 und den beschränkten Regelkreis ohne Antiwindup (o. A.) aus Bild 2.

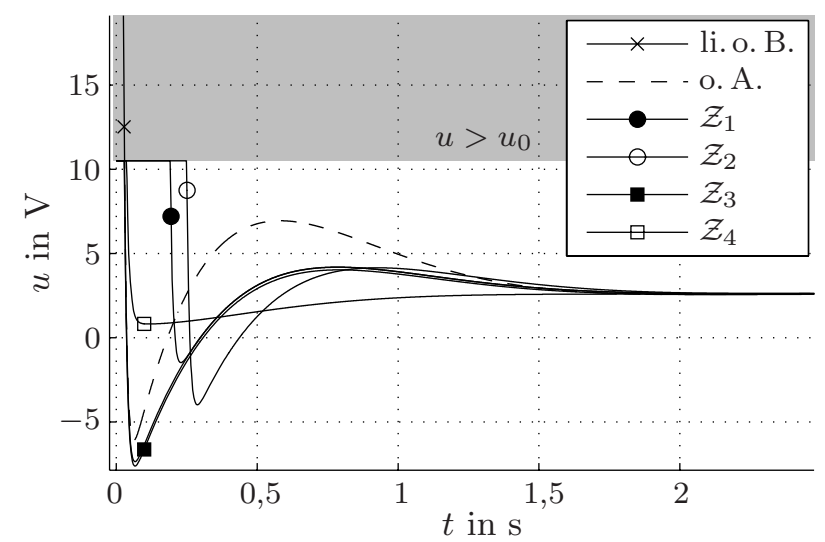

Bild 11 Stellgröße $u$ für $\mathcal{Z}_{1}$ bis $\mathcal{Z}_{4}$, das lineare geregelte System ohne Beschränkung (li.o. B.) und den beschränkten Regelkreis ohne Antiwindup (o. A.). Der graue Bereich kennzeichnet die in den Regelkreisen mit Beschränkung nicht realisierbaren Stellgrößen $u>10,5$ V. Die Zeitachse weist eine andere Skalierung auf.

Schritt 2: Mit $K=1$ ist eine Abschätzung der $L_{2}$ Verstärkung nicht für alle $\mathcal{Z} \in M_{\mathcal{Z}}^{\text {stabil }}$ möglich, da das Optimierungsproblem (41) nicht immer eine Lösung liefert. Deshalb wird Schritt 2 mit $K<1$ wiederholt. Für $K=0,97$ lässt sich für alle $\mathcal{Z} \in M_{\mathcal{Z}}^{\text {stabil }}$ eine $L_{2}$ Kleinsignalverstärkung $\gamma$ abschätzen. Die Ergebnisse sind ebenfalls in Tabelle 1 aufgelistet. Da $\mathcal{Z}_{2}$ die kleinste Verstärkung garantiert, wird diese Zerlegung gewählt.

Die Simulationsergebnisse in Bild 10 und Bild 11 bestätigen, dass ein mit $\mathcal{Z}_{2}=\left\{\Delta_{2}(s), R_{2}(s)\right\}$ realisiertes Regler-Antiwindup am nächsten an das erwünschte lineare Reglerverhalten, das die Stellgröße weit über den erlaubten Bereich $\left[-u_{0}, u_{0}\right]$ nutzt, heranreicht.

\section{Zusammenfassung}

In diesem Beitrag wurde ein systematisches Auswahlverfahren für das Regler-Antiwindup nach Hippe vorgestellt, das die Problematik der Nullstellenwahl für das Polynom $\Delta(s)$ löst. Das zweistufige Verfahren kombiniert ein einfach anwendbares Stabilitätskriterium mit einer auf konvexer Optimierung basierenden Abschätzung der Regelgüte. Für jede Reglerzerlegung wird ein Kennwert berechnet, der ein Maß für die erreichbare Regelgüte darstellt. Eine Evaluation sämtlicher Zerlegungen anhand von Simulationen ist nicht mehr nötig, da die Auswahl anhand des Kennwertes erfolgt. Am Beispiel der Regelung eines hydraulischen Aktors wurde das Verfahren demonstriert.

\section{A Anhang}

Es wird gezeigt, dass das Ein-/Ausgangsverhalten der $\mathrm{Zu}$ standsraumdarstellung (23) mit $\boldsymbol{f}$ gemäß Gl. (25) dem nichtlinearen Teil der Mismatch-Darstellung aus Bild 6 entspricht. Das ist erfüllt, wenn

$$
\frac{Y_{d}(s)}{\tilde{U}(s)}=\frac{Z(s)}{R(s)} \quad \text { und } \quad \frac{U_{d}(s)}{\tilde{U}(s)}=\frac{N(s)-R(s)}{R(s)}
$$

gilt. Das System (23) lässt sich mit $\tilde{u}=\operatorname{dzn}\left(u_{\text {lin }}-u_{d}\right)$ auch darstellen als

$$
\begin{aligned}
& \dot{z}=\boldsymbol{A}_{f} z+\boldsymbol{b} \tilde{u}=\left(\boldsymbol{A}+\boldsymbol{b} \boldsymbol{f}^{\top}\right) z+\boldsymbol{b} \tilde{u}, \\
& y_{d}=\boldsymbol{c}^{\top} z, \\
& u_{d}=\boldsymbol{f}^{\top} z .
\end{aligned}
$$

Die Strecke (1) mit den Systemmatrizen $\boldsymbol{A}, \boldsymbol{b}, \boldsymbol{c}$ liegt in Regelungsnormalform (RNF) vor und das $\operatorname{Paar}(\boldsymbol{A}, \boldsymbol{b})$ ist vollständig steuerbar. Daraus folgt:

1. Die Elemente in der $n$-ten Zeile der Matrix $\boldsymbol{A}$ sind $\left[-a_{0} \ldots-a_{n-1}\right]$ und entsprechen den negativen Koeffizienten des Nennerpolynoms der Strecke, d.h. $N(s)=s^{n}+a_{n-1} s^{n-1}+\ldots+a_{0}$.

2. Es lässt sich immer ein $\boldsymbol{f}=\left[f_{1} \ldots f_{n}\right]$ finden, sodass

$$
\begin{aligned}
R(s) & =\operatorname{det}\left(s \boldsymbol{I}-\left(\boldsymbol{A}+\boldsymbol{b} \boldsymbol{f}^{\boldsymbol{\top}}\right)\right)=\operatorname{det}\left(s \boldsymbol{I}-\boldsymbol{A}_{f}\right) \\
& =s^{n}+\left(a_{n-1}-f_{n}\right) s^{n-1}+\ldots+\left(a_{0}-f_{1}\right) .
\end{aligned}
$$

3. Die Elemente des Vektors $\boldsymbol{c}^{\top}=\left[\begin{array}{llll}c_{0} & \ldots & c_{n-1}\end{array}\right]$ sind die Koeffizienten des Zählerpolynoms der Strecke, d.h. $Z(s)=c_{n-1} s^{n-1}+\ldots+c_{0}$.

4. Aus Punkt 1 und Punkt 2 folgt: Die Elemente des Vektors $\boldsymbol{f}$ entsprechen den Koeffizienten des Polynoms $N(s)-R(s)=f_{n} s^{n-1}+\ldots+f_{1}$. 
Das Übertragungsverhalten von $\tilde{u}$ nach $y_{d}$ wird durch die Gleichungen (A.1a), (A.1b) bestimmt. Dieses System liegt in RNF vor und besitzt die Übertragungsfunktion

$\frac{Y_{d}(s)}{\tilde{U}(s)}=\frac{c_{n-1} s^{n-1}+\ldots+c_{0}}{\operatorname{det}\left(s \boldsymbol{I}-\boldsymbol{A}_{f}\right)}=\frac{Z(s)}{R(s)}$.

Das Übertragungsverhalten von $\tilde{u}$ nach $u_{d}$ wird durch die Gleichungen (A.1a), (A.1c) bstimmt. Dieses System liegt ebenfalls in RNF vor und besitzt die Übertragungsfunktion

$$
\frac{U_{d}(s)}{\tilde{U}(s)}=\frac{f_{n-1} s^{n-1}+\ldots+f_{0}}{\operatorname{det}\left(s \boldsymbol{I}-\boldsymbol{A}_{f}\right)}=\frac{N(s)-R(s)}{R(s)} .
$$

\section{Literatur}

[1] Adamy, J.: Nichtlineare Regelungen. Springer, 2009.

[2] Boyd, S., L. E. Ghaoui, E. Feron und V. BalakrishNAN: Linear matrix inequalities in system and control theory. SIAM, 1994.

[3] Ebenbauer, C., T. Raff und F. Allgöwer: Dissipation inequalities in systems theory: An introduction and recent results. In: 6th International Conference on Industrial and Applied Mathematics, Zürich, Schweiz, 2007.

[4] Hippe, P.: Eine neue Methode zur Regelung von Strecken mit Stellbegrenzung. Automatisierungstechnik, 52(9):421-431, 2004.

[5] Hippe, P.: Windup in Control - Its Effects and Their Prevention. Springer, 2006.

[6] Hippe, P., C. Wurmthaler, A. H. Glattfelder und W. Schaufel Berger: Regelung von Strecken mit Stellbegrenzung. In: ENGELL, S. (Hrsg.): Entwurf nichtlinearer Regelungen, S. 239-264, Oldenbourg, 1995.

[7] Kothare, M. V., P. J. Campo, M. Morari und C. N. Netт: A unified framework for the study of anti-windup designs. Automatica, 30(12):1869-1883, 1994.

[8] Kugi, A. und K. Schlacher: Analyse und Synthese nichtlinearer dissipativer Systeme: Ein Überblick (Teil 1). Automatisierungstechnik, 50(2):63-69, 2002.

[9] Lens, H. und J. Adamy: Weiche strukturvariable Regelungen mit Beobachtern für einen hydraulischen Aktor. Automatisierungstechnik, 55(8):411-417, 2007.

[10] Lunze, J.: Regelungstechnik 2. Springer, 2008.

[11] LÖFBERG, J.: YALMIP: a toolbox for modeling and optimization in MATLAB. In: International Symposium on Computer Aided Control Systems Design, S. 284-289, Taipeh, Taiwan, 2004.
[12] Narendra, K. S. und J. H. TAYLOR: Frequency Domain Criteria for Absolute Stability. Academic Press, 1973.

[13] Reinschke, K.: Lineare Regelungs- und Steuerungstheorie. Springer, 2005.

[14] Skogestad, S. und I. Postlethwaite: Multivariable Feedback Control: Analysis and Design. Wiley-Interscience, 2005.

[15] Sofrony, J., M. Turner und I. Postlethwaite: Antiwindup synthesis using Riccati equations. International Journal of Control, 80(1):112-128, 2007.

[16] Tarbouriech, S. und M. Turner: Anti-windup design: an overview of some recent advances and open problems. IET Control Theory and Applications, 3(1):1-19, 2009.

[17] Toh, K.-C., M. J. Todd und R. H. Tutuncu: SDPT3 A Matlab software package for semidefinite programming. Optimization Methods and Software, 11(1-4):545-581, 1999.

[18] Turner, M. C., G. Herrmann und I. Postlethwaite: Anti-windup compensation using a decoupling architecture. In: TArbouriech, S. (Hrsg.): Advanced Strategies in Control Systems with Input and Output constraints, S. 121-171. Springer, 2007.

[19] Weston, F. und I. Postlethwaite: Linear conditioning for systems containing saturating actuators. Automatica, 36(9):1347$1354,2000$.

Manuskripteingang: 28. Juni 2010

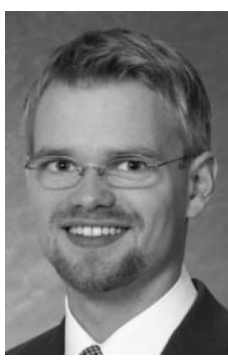

Dipl.-Ing. Andreas Ortseifen promoviert am Fachgebiet Regelungstheorie und Robotik der TU Darmstadt im Bereich nichtlineare Regelungen. Hauptarbeitsgebiete: Antiwindup, Systeme mit Stellgrößenbeschränkungen.

Adresse: Technische Universität Darmstadt, FB Elektrotechnik und Informationstechnik, Fachgebiet Regelungstheorie und Robotik, LandgrafGeorg-Str. 4, D-64283 Darmstadt,

E-Mail: ortseifen@rtr.tu-darmstadt.de

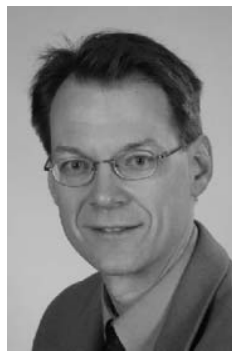

Prof. Dr.-Ing. Jürgen Adamy ist Leiter des Fachgebietes Regelungstheorie und Robotik im Fachbereich Elektrotechnik und Informationstechnik der TU Darmstadt. Hauptarbeitsgebiete: Regelungsverfahren, Computational Intelligence, autonome mobile Roboter.

Adresse: Technische Universität Darmstadt, FB Elektrotechnik und Informationstechnik, Fachgebiet Regelungstheorie und Robotik, LandgrafGeorg-Str. 4, D-64283 Darmstadt,

E-Mail: adamy@rtr.tu-darmstadt.de 